\title{
An examination of factors influencing the choice of therapy for patients with coronary artery disease Shashivadan P Hirani ${ }^{1}$, Jonathan A Hyam ${ }^{1}$, Shahzad Shaefi ${ }^{1}$, John M Walker ${ }^{2}$, Robin K Walesby ${ }^{3}$ and Stanton P Newman*1
}

Address: ${ }^{1}$ Health Psychology Unit, Centre for Behavioural and Social Sciences in Medicine University College London, Wolfson Building, 48 Riding House Street, London W1W 7EY, UK, ${ }^{2}$ Centre for Cardiology and The Hatter Institute for Cardiovascular Studies University College London Hospital, Grafton Way, London WC1E 6DB, UK and ${ }^{3}$ The Heart Hospital University College London Hospital, 16 Westmoreland Street, London W1G 8PH, UK

Email: Shashivadan P Hirani - s.hirani@ucl.ac.uk; Jonathan A Hyam - jhyam25@hotmail.com; Shahzad Shaefi - sshaefi@partners.org; John M Walker - malcolm.walker@uclh.org; Robin K Walesby - robin.walesby@uclh.org; Stanton P Newman* - s.newman@ucl.ac.uk

* Corresponding author

Published: 04 July 2006

BMC Cardiovascular Disorders 2006, 6:31 doi:|0.1186/|47|-226I-6-31
Received: 20 December 2005

Accepted: 04 July 2006

This article is available from: http://www.biomedcentral.com/l47I-226I/6/3I

(C) 2006 Hirani et al; licensee BioMed Central Ltd.

This is an Open Access article distributed under the terms of the Creative Commons Attribution License (http://creativecommons.org/licenses/by/2.0), which permits unrestricted use, distribution, and reproduction in any medium, provided the original work is properly cited.

\begin{abstract}
Background: A diverse range of factors influence clinicians' decisions regarding the allocation of patients to different treatments for coronary artery disease in routine cardiology clinics. These include demographic measures, risk factors, co-morbidities, measures of objective cardiac disease, symptom reports and functional limitations. This study examined which of these factors differentiated patients receiving angioplasty from medication; bypass surgery from medication; and bypass surgery from angioplasty.
\end{abstract}

Methods: Univariate and multivariate logistic regression analyses were conducted on patient data from 214 coronary artery disease patients who at the time of recruitment had been received a clinical assessment and were reviewed by their cardiologist in order to determine the form of treatment they were to undergo: 70 would receive/continue medication, 71 were to undergo angioplasty and 73 were to undergo bypass surgery.

Results: Analyses differentiating patients receiving angioplasty from medication produced 9 significant univariate predictors, of which 5 were also multivariately significant (left anterior descending artery disease, previous coronary interventions, age, hypertension and frequency of angina). The analyses differentiating patients receiving surgery from angioplasty produced 12 significant univariate predictors, of which 4 were multivariately significant (limitations in mobility range, circumflex artery disease, previous coronary interventions and educational level). The analyses differentiating patients receiving surgery from medication produced 14 significant univariate predictors, of which 4 were multivariately significant (left anterior descending artery disease, previous cerebral events, limitations in mobility range and circumflex artery disease).

Conclusion: Variables emphasised in clinical guidelines are clearly involved in coronary artery disease treatment decisions. However, variables beyond these may also be important factors when therapy decisions are undertaken thus their roles require further investigation. 


\section{Background}

Coronary artery disease (CAD) in the United Kingdom accounted for over 117,000 deaths during 2002 and continues to represent a common cause of mortality in the UK and in most developed societies [1]. Current management options for CAD fall into three main categories, namely medical therapy, percutaneous transluminal coronary angioplasty with or without stenting, (PTCA) and coronary artery bypass grafting (CABG). In the UK in the National Health Service up to 45,000 PTCA and 28,500 CABG are conducted annually; each at a current average cost of $£ 2,609(\sim \$ 4,500)$ and $£ 7,066(\sim \$ 12,000)$ respectively [2]. Where detailed guidelines on the use of PTCA and CABG have been proposed, they address a myriad of precise individual scenarios and are considered extremely complex [3-8]. Many parameters such as the variability of symptoms and employment status are not addressed in guidelines despite being considered to have an important role in decision making and being routinely addressed by clinicians. Rapid innovations in treatment often leave guidelines struggling to keep pace with current practice. In this context, the advent of minimally invasive options for surgery, advanced percutaneous techniques and improved medication regimens alter the risk benefit ratio and often result in guidelines lagging behind with technological advances that are already in clinical use.

A range of studies have reported that a not insignificant proportion of PTCAs and, to a lesser degree, CABGs are performed inappropriately or with uncertain appropriateness [9-11]. These figures vary according to location (e.g. US data indicates overuse of revascularization and UK data its under use), the reference guidelines utilised and the recency of the update of the guidelines. The allocation of patients to particular therapies appears to be influenced by a range of factors beyond guidelines and recommendations.

The aim of this paper was to examine the factors that influence clinical decisions regarding the allocation of patients to different treatments for coronary artery disease in routine cardiology clinics. It was hypothesised that clinicians would use both factors within guidelines as well as those outside the guidelines. Separate analyses were conducted to compare pairs of treatments from medication, PTCA and CABG, to enable stepwise analyses to be conducted that could determine the 'best' predictor variables. It was expected that for the more disparate interventions (e.g. medication and CABG), objective criteria would be likely predictors of treatment choice; but when interventions were less disparate (e.g. PTCA vs. CABG or medication vs. PTCA), variables beyond those in the guidelines would be likely to exert a predictive influence.

\section{Methods \\ Patient population}

Between January 1999 and February 2001, 294 patients with CAD from the caseloads of seven cardiologists, from three National Health Service centres in London and surrounding areas were selected as part of a convenience sample. The participants were invited to take part in the study in person or by mail. The patients were approached after their cardiologist/consultant determined the form of treatment they were to undergo, following a review of clinical data (including angiograms) and patient consultations. The clinician determined whether the patients were to start/continue medication or undergo revascularisation by PTCA or CABG surgery. Two-hundred and fourteen patients $(72.79 \%)$ consented to take part in the study. The response rate per treatment was similar, with $73.00 \%$ of those about to undergo CABG, $74.74 \%$ of those about to undergo PTCA and $70.71 \%$ of those to receive medication consenting; with the resulting sample consisting of 70 in receipt of medication, 71 to receive PTCA and 73 to receive $C A B G$ surgery.

\section{Measures}

Objective disease measures (angiogram data) were retrieved from patient medical records. Demographic, medical history, subjective symptoms and functional limitations data were obtained from patient self-reported responses to a questionnaire pack collated for the study. Demographic details included gender, age, employment situation, and educational level (scored on a scale from 1 - no formal education after compulsory level, to 4 - graduate/professional exams and above).

Previous cardiac history (previous interventions, previous myocardial infarction), coronary risk factors (hypercholesterolemia, hypertension, diabetes, smoking history and family history for myocardial infarction, ischaemic heart disease, stroke and neurological conditions) and co-morbidities (arthritis/rheumatism, respiratory disease, renal complications, cerebral events, gastrointestinal, peripheral vascular problems, thyroid problems and varicose veins) were measured as binary yes/no responses, except for smoking history which had three categories: never smoked, ex-smoker and still smoking.

Symptom reports were elicited for angina, dyspnoea and for a range of non-cardiac symptoms. Two items asked patients the frequency with which they currently experienced chest pains and shortness of breath. A further 31 items assessed a range of general symptoms (e.g. fatigue, sleep difficulties, muscle pains, vomiting) from which an average non-cardiac symptom-experience score was produced. Responses for all these items were recorded on a 4point scale, from all the time to never, with higher scores representing greater frequency. 
Scores for functional limitations due to cardiac symptoms were collected through scales adapted from Feinstein ratings [12]. These scales provided two scores relating to the level of functional impairment resultant from (i) angina and (ii) dyspnoea on the dimensions of task, pace and daily functions. Averaged impairment scores ranged from 0 to 4 ; higher scores represented greater limitations.

Further health related functional disability scores were obtained from the six subscales of the Sickness Impact Profile-68 (SIP) [13]. The scales were: 'social behaviour' (scores ranging from 0 to 12$)$, 'mobility range' (0 to 10$)$, 'emotional stability' (0 to 6$)$,'somatic autonomy' ( 0 to 17 ), 'mobility control' ( 0 to 12 ) and 'psychological autonomy \& communication' (0 to 11 ), with higher scores representing greater limitations in daily functioning.

An objective measure of disease severity was obtained from angiograms. These were analysed for the level of disease in three coronary disease prone arteries: the left anterior descending, the circumflex and right coronary arteries, each of which was rated on a four point scale (no significant disease, mild, moderate or severe). Additional measures of left ventricular function (no significant problems, mild, moderate or severe problems) and the number of diseased arteries were recorded, providing a total of five scores. These ratings were based on the agreed ratings of two cardiologists.

The majority of patients $(86.9 \%-186 / 214)$ patients were administered the questionnaires by one of the authors (SPH) at the bedside or in home interviews. However due to logistical reasons 5 medication and 23 PTCA patients completed the questionnaires by themselves and posted them back to the researchers.

Ethical approval for this study was granted by: Research and Development Directorate of University College London Hospital NHS Trust (ref: 99/0059) and the Clinical Research, Education, Effectiveness and Development Local Research and Ethics Committee of the Whittington Hospital (ref: 99/05L).

\section{Statistical analyses}

The SPSS for Windows 11, LISREL 8.52 and SAS v8.2 statistical software packages were used for the analyses.

Preliminary examinations of the data revealed that the overall level of missing data was $3.43 \%$ (279 scores from 8132 items). Nevertheless, sixty cases (28.04\%) had data missing on at least one variable, indicating that although the overall level of missing data is low, its effects can be relatively large. Missing values were imputed using the Markov Chain Monte Carlo algorithm through LISREL after finding no statistically reliable deviation from ran- domness using Little's MCAR test: $\chi^{2}=80.272, \mathrm{df}=96, \mathrm{p}$ $=0.876$ (within SPSS 11). The remaining analyses were conducted on the imputed data set $[14,15]$.

Differences between treatment groups on each predictor variable was tested using the $\chi^{2}$ test and one-way ANOVA's (Brown-Forsythe test when unequal variances) with post hoc Duncan's multiple range tests. Significance levels were set at $\mathrm{p}<0.01$. Where appropriate, measures of the strength of association between variables are provided through omega squared $\left(\omega^{2}\right)$ and Cramer's phi $\left(\phi_{c}\right)$, also see Table 1.

Thereafter, three separate sets of logistic regression analyses were conducted with the same analytic procedures. In each analysis the likelihood of a particular treatment option (response category) was compared to a less invasive procedure (the reference category). The analyses performed were: (i) PTCA vs. medication, (ii) CABG vs. PTCA, and (iii) CABG vs. medication.

A series of univariate logistic analyses were conducted to identify individually significant predictors of the response treatment from the reference treatment in each of the three analyses. The likelihood ratio criterion was employed to test for the significance $(p<0.05)$ of each predictor. Variables with $\mathrm{p}<0.05$ within the univariate analyses were entered into a stepwise multivariate logistic analysis, to identify independent predictors of treatment type in each analysis. Significance was set at $p<0.01$ in these multivariate logistic regressions. Variables within the regression equation were analyzed for removal at each step, using a p > 0.05 significance level. The stepwise process was halted when the residual chi-square was no longer significant.

The final model was evaluated for, (i) goodness of fit (deviance statistic), (ii) the association of predictor variables with the outcome variable (Nagelkerke's $\mathrm{R}^{2}$ ), (iii) success in classification (\% correctly classified cases, sensitivity and specificity), and (iv) the discriminatory power of the logistic equation ( $c$ statistic), see Table 1.

Individual parameters in each model are presented as odds ratios for categorical data and p-deviance for continuous variables. To aid interpretation, on occasions when the odds ratio was less than one, its reciprocal was taken and the finding interpreted in terms of the odds regarding the reference group.

\section{Results \\ Preliminary group differences \\ Demographic factors}

The sample consisted of 175 males and 39 females, with similar proportions of males and females in each treat- 
Table I: Criteria for statistical interpretation

\begin{tabular}{|c|c|c|c|}
\hline & Statistic & & Interpretation \\
\hline \multirow[t]{6}{*}{ Strength of association } & $\omega^{2}$ & $\approx 0.01$ & small association \\
\hline & & $\approx 0.06$ & medium association \\
\hline & & $\approx 0.14$ & large association \\
\hline & $\phi_{c}$ & $<0.3$ & little or no association \\
\hline & & 0.3 to 0.7 & weak association \\
\hline & & 0.7 to 1.0 & strong association \\
\hline \multirow[t]{6}{*}{ Regression model evaluation } & deviance statistic & - & $\begin{array}{l}\text { significance of unexplained (residual) variance in the dependent variable; } \\
\text { thus is desired to be non-significant }\end{array}$ \\
\hline & Nagelkerke's R² & - & $\begin{array}{l}\text { the association between predictor variables and the outcome variable. } \\
\text { Statistic ranges from } 0 \text { to I with higher scores indicating stronger } \\
\text { associations. }\end{array}$ \\
\hline & $\%$ of correctly classified cases & - & $\begin{array}{l}\text { calculated at the start of the procedure and at the end of the analysis } \\
\text { using the cut-off point of } 0.5 \text { for the estimated probability of the } \\
\text { outcome variable }\end{array}$ \\
\hline & sensitivity & - & $\begin{array}{l}\text { the proportion of true positives or the proportion of cases correctly } \\
\text { identified by the test as meeting the response category }\end{array}$ \\
\hline & specificity & - & $\begin{array}{l}\text { the proportion of true negatives or the proportion of cases correctly } \\
\text { identified by the test as meeting the reference category }\end{array}$ \\
\hline & c statistic & - & $\begin{array}{l}\text { varies from } 0.5 \text { (the models predictions are no better than chance) to I } \\
\text { (the model always assigns the higher probabilities to correct cases than } \\
\text { incorrect ones) }\end{array}$ \\
\hline
\end{tabular}

ment group (CABG: 66 males, 7 females; PTCA: 57 males, 14 females; medication: 52 males, 18 females; $\chi^{2}=6.394$, $\mathrm{df}=2, \mathrm{p}=0.041 ; \phi_{\mathrm{c}}=0.173$ ). The mean age of the sample was 64.1 years, with no age differences between treatment groups $\left(\mathrm{f}_{(2,213)}=2.588, \mathrm{p}=0.078 ; \omega^{2}=0.015\right)$. The employment status of patients did not significantly differ between groups $\left(\chi^{2}=19.967, \mathrm{df}=12, \mathrm{p}=0.068 ; \phi_{\mathrm{c}}=\right.$ 0.216). The PTCA participants were found to have a higher educational level than the other two groups (Brown-Forsythe, $\mathrm{f}_{(2,202.030)}=10.605, \mathrm{p}<0.001 ; \omega^{2}=$ 0.082 ; PTCA mean $=2.51$, medication mean $=1.97$, CABG mean score $=1.64$ ).

\section{Cardiac history, risk factors and co-morbidities}

The three treatment groups were relatively similar with regards co-morbidity, risk factors and cardiac history. Only three variables differed significantly between the groups. The medication group had the highest rate of cerebral events $(\mathrm{n}=12)$, followed by the PTCA $(\mathrm{n}=7)$ then the CABG $\left(\mathrm{n}=1 ; \chi^{2}=10.526, \mathrm{df}=2, \mathrm{p}=0.005 ; \phi_{\mathrm{c}}=\right.$ 0.222 ); however, the overall frequency of events was modest. The PTCA group had more previous interventions than the other two groups $\left(\chi^{2}=27.779, \mathrm{df}=2, \mathrm{p}<0.001\right.$; $\left.\phi_{c}=0.360\right)$, the majority of which were previous PTCA (13/25 PTCA alone; 8/25 CABG alone; 4/25 PTCA and CABG). Rates of family history of IHD also varied significantly between groups $\left(\chi^{2}=11.393, \mathrm{df}=2, \mathrm{p}=0.003 ; \phi_{\mathrm{c}}=\right.$ $0.231)$. PTCA $(\mathrm{n}=34)$ patients had lower rates than the medication $(n=51)$ and CABG $(n=51)$ groups. Rates of other conditions were similar between treatments groups, and the common risk factors for CAD did not significantly differ between groups.

\section{Angiogram reports}

Objective disease scores revealed significant treatment group effects $(\mathrm{p}<0.01)$ for all indices except right coronary artery disease $\left(\mathrm{f}_{(2,111)}=4.319, \mathrm{p}=0.015 ; \omega^{2}=0.030\right)$. LAD artery disease (Brown-Forsythe, $\mathrm{f}_{(2,184.061)}=31.712$, $\mathrm{p}$ $\left.<0.001 ; \omega^{2}=0.223\right)$, significantly differed between medication and the two revascularisation groups. Circumflex artery scores (Brown-Forsythe, $\mathrm{f}_{(2,201.629)}=19.785, \mathrm{p}<$ $0.001 ; \omega^{2}=0.149$ ), total vessels diseased scores (BrownForsythe, $\left.\mathrm{f}_{(2,183.301)}=6.525, \mathrm{p}=0.002 ; \omega^{2}=0.049\right)$, and ventricular function scores (Brown-Forsythe, $\mathrm{f}_{(2,183.046)}=$ 8.533, $\left.\mathrm{p}<0.001 ; \omega^{2}=0.066\right)$, all produced a pattern of group differences where the medication and PTCA groups significantly differing from the CABG group but not from each other. Group mean scores are shown in Table 2.

\section{Cardiac symptoms and associated functional limitations}

Significant treatment group differences were found on the angina frequency scores, $\left(f_{(2,211)}=5.444, p=0.005 ; \omega^{2}=\right.$ $0.040)$. Post hoc tests revealed the medication group to have significantly less frequent angina than the PTCA and CABG groups. The frequency of shortness of breath did not significantly vary between treatment groups $\left(\mathrm{f}_{(2,211)}=\right.$ $\left.1.562, \mathrm{p}=0.212 ; \omega^{2}=0.005\right)$; nor did the level of non-cardiac symptoms $\left(f_{(2,211)}=1.640, p=0.196 ; \omega^{2}=0.006\right)$. 
Table 2: Demographics, angiogram scores, symptom reports and functional ability by treatment group

\begin{tabular}{|c|c|c|c|c|c|c|}
\hline & & Total & $\begin{array}{l}\text { Medicati } \\
\text { on }\end{array}$ & PTCA & CABG & $P$ \\
\hline & & 214 & 70 & 71 & 73 & \\
\hline \multirow[t]{2}{*}{ Gender } & Male & 175 & 52 & 57 & 66 & \\
\hline & Female & 39 & 18 & 14 & 7 & 0.041 \\
\hline Age & Mean & 64.09 & 66.00 & 62.03 & 64.26 & 0.078 \\
\hline Education level & Mean & 2.04 & $1.97^{a}$ & $2.5 \mathrm{I}^{\mathrm{b}}$ & $1.64^{\mathrm{a}}$ & $<0.001$ \\
\hline \multirow[t]{5}{*}{ Angiogram scores } & LAD artery disease & 2.18 & $1.56^{\mathrm{a}}$ & $2.3 \mathrm{I}^{\mathrm{b}}$ & $2.64 b$ & $<0.001$ \\
\hline & Circumflex artery disease & 1.67 & $1.30^{\mathrm{a}}$ & $1.39 \mathrm{a}$ & $2.30^{\mathrm{b}}$ & $<0.001$ \\
\hline & RCA disease & 1.98 & 1.80 & 1.85 & 2.27 & 0.015 \\
\hline & Number of disease vessels & 2.51 & $2.39^{a}$ & $2.37^{a}$ & $2.77^{b}$ & 0.002 \\
\hline & Ventricular Function score & 0.63 & $0.56^{\mathrm{a}}$ & $0.39 a$ & $0.93^{b}$ & $<0.001$ \\
\hline \multirow[t]{3}{*}{ Symptom frequency } & Angina & 2.03 & $1.80^{a}$ & $2.18^{\mathrm{b}}$ & $2.11^{b}$ & 0.005 \\
\hline & Dyspnoea & 2.22 & 2.19 & 2.37 & 2.11 & 0.212 \\
\hline & Non-cardiac & 1.43 & 1.38 & 1.46 & 1.46 & 0.196 \\
\hline \multirow[t]{2}{*}{ Functional limitations } & Angina associated & 1.80 & $1.29 \mathrm{a}$ & $1.93^{b}$ & $2.15^{b}$ & $<0.001$ \\
\hline & Dyspnoea associated & 1.56 & 1.21 & 1.67 & 1.79 & 0.013 \\
\hline \multirow[t]{6}{*}{ Activities of daily living } & Social Behaviour & 4.43 & $3.96^{a}$ & $3.97^{a}$ & $5.32^{\mathrm{b}}$ & 0.005 \\
\hline & Emotional Stability & 1.30 & 1.43 & 1.48 & 1.00 & 0.112 \\
\hline & Mobility Range & 1.91 & $1.00^{a}$ & $1.15^{a}$ & $3.52^{\mathrm{b}}$ & $<0.001$ \\
\hline & Somatic Autonomy & 0.84 & 0.94 & 0.58 & 0.99 & 0.200 \\
\hline & Mobility Control & 4.00 & 3.81 & 3.63 & 4.53 & 0.143 \\
\hline & Psychological Autonomy \& Communication & 1.71 & 1.89 & 1.75 & 1.51 & 0.613 \\
\hline
\end{tabular}

Gender analyses conducted with Chi-sq test, remainder with ANOVAs.

Significant treatment group differences revealed within the ANOVAs are indicated with different superscript letters.

One-way ANOVAs of the functional limitations associated with cardiac symptoms, revealed that the ratings for angina were significantly different between treatment groups (Brown-Forsythe, $\mathrm{f}_{(2,204.159)}=9.618, \mathrm{p}<0.001 ; \omega^{2}$ $=0.075$ ), but not for dyspnoea (Brown-Forsythe, $\left.\mathrm{f}_{(2,201.311)}=4.474, \mathrm{p}=0.013 ; \omega^{2}=0.031\right)$. The post hoc tests for angina ratings showed the medication group was significantly less functionally impaired by angina than both the PTCA and CABG patients.

\section{Activities of daily living}

On limitations in activities of daily living, the groups differed significantly on two indices, social behaviour $\left(f_{(2,211)}=5.398, p=0.005 ; \omega^{2}=0.039\right)$ and mobility range (Brown-Forsythe, $\mathrm{f}_{(2,185.686)}=33.072, \mathrm{p}<0.001 ; \omega^{2}=$ $0.231)$. In both cases, the medication and PTCA groups were significantly different from the CABG group but not from each other. The other activity indices revealed similar group scores: Emotional Stability, (Brown-Forsythe, $\left.\mathrm{f}_{(2,197.489)}=2.211, \mathrm{p}=0.112 ; \omega^{2}=0.011\right)$; Somatic Autonomy, $\left(\mathrm{f}_{(2,211)}=1.612, \mathrm{p}=0.200 ; \omega^{2}=0.006\right)$; Mobility Control, $\left(f_{(2,211)}=1.963, p=0.143 ; \omega^{2}=0.009\right)$; and Psy- chological Autonomy and Communication, $\left(\mathrm{f}_{(2,211)}=\right.$ $\left.0.490, \mathrm{p}=0.613 ; \omega^{2}=0.000\right)$.

\section{Predictors of treatment group}

The univariate analyses (Table 3 ) were followed by the same comparisons in a multivariate analysis (Table 4). The results of both sets of analyses are collated and summarised in Table 5.

\section{Analyses I: PTCA compared to medication}

In the univariate analysis, nine of the predictor variables produced significant findings (see Table 3a). Increasing age, hypertension and a family history of ischemic heart disease (IHD) reduced the odds of a patient receiving PTCA. Educational level, previous interventions, angina and dyspnoea related functional limitations, angina frequency and LAD disease, increased the odds of PTCA.

Within the multivariate analysis (Table 4a), LAD artery disease, previous intervention, age, hypertension and angina frequency were included in the final model. The overall model produces a satisfactory goodness of fit, with non-significant residual variance $($ deviance $=135.184, \mathrm{df}$ 
Table 3: Variables that achieved significance in the univariate comparisons between treatment groups

\begin{tabular}{|c|c|c|c|c|c|c|c|c|}
\hline \multirow[t]{2}{*}{ VARIABLES } & \multicolumn{3}{|c|}{ Likelihood ratio } & \multirow{2}{*}{$\begin{array}{c}\operatorname{Exp}(\beta) \\
\text { Odds Ratio }\end{array}$} & \multicolumn{2}{|c|}{ Confidence interval } & \multicolumn{2}{|c|}{ RATES/MEAN SCORES } \\
\hline & Model change & df. & Sig. & & Lower bound & Upper bound & Reference & Response \\
\hline (a) PTCA from Medication & & & & & & & Medication & PTCA \\
\hline Increasing age & 4.644 & 1 & 0.031 & 0.967 & 0.928 & 1.008 & 66.00 & 62.03 \\
\hline Higher educational attainment & 6.887 & 1 & 0.009 & 1.447 & 0.999 & 2.096 & 1.97 & 2.51 \\
\hline Previous intervention & 20.517 & 1 & $<0.001$ & 8.961 & 2.055 & 39.067 & 4 & 25 \\
\hline Hypertension & 4.505 & 1 & 0.034 & 0.482 & 0.197 & 1.178 & 36 & 24 \\
\hline Family history: IHD & 9.306 & 1 & 0.002 & 0.342 & 0.136 & 0.863 & 51 & 34 \\
\hline LAD artery disease & 21.166 & 1 & $<0.001$ & 2.328 & 1.392 & 3.893 & 1.56 & 2.31 \\
\hline Angina frequency & 11.204 & 1 & 0.001 & 2.351 & $\mathrm{I} .182$ & 4.677 & 1.80 & 2.18 \\
\hline Angina functional limitations & 11.079 & 1 & 0.001 & 1.651 & 1.102 & 2.474 & 1.29 & 1.93 \\
\hline Dyspnoea functional limitations & 5.851 & 1 & 0.016 & 1.444 & 0.968 & 2.153 & 1.21 & 1.67 \\
\hline (b) CABG from PTCA & & & & & & & PTCA & CABG \\
\hline Higher educational attainment & 19.186 & 1 & $<0.001$ & 0.525 & 0.350 & 0.786 & 2.51 & 1.64 \\
\hline Previous intervention & 16.410 & 1 & $<0.001$ & 0.165 & 0.046 & 0.587 & 25 & 6 \\
\hline Family history: IHD & 7.251 & 1 & 0.007 & 2.523 & 1.028 & 6.192 & 34 & 51 \\
\hline Family history: Neurological & 5.400 & 1 & 0.020 & 0.194 & 0.025 & 1.527 & 9 & 2 \\
\hline Cerebral events & 5.505 & 1 & 0.019 & 0.127 & 0.008 & 2.065 & 7 & I \\
\hline LAD artery disease & 6.996 & 1 & 0.008 & $\mathrm{I} .834$ & 0.980 & 3.435 & 2.31 & 2.64 \\
\hline Circumflex artery disease & 22.489 & 1 & $<0.001$ & 2.058 & 1.352 & 3.132 & 1.39 & 2.30 \\
\hline $\mathrm{RCA}$ disease & 5.600 & 1 & 0.018 & 1.444 & 0.960 & 2.173 & 1.85 & 2.27 \\
\hline Number of vessels diseased & 12.709 & 1 & $<0.001$ & 2.546 & 1.221 & 5.310 & 2.37 & 2.77 \\
\hline Ventricular disease & 12.979 & 1 & $<0.001$ & 2.037 & 1.172 & 3.540 & 0.39 & 0.93 \\
\hline SIP: Social behaviour & 7.937 & 1 & 0.005 & 1.182 & 1.010 & 1.382 & 3.97 & 5.32 \\
\hline SIP: Mobility range & 35.312 & 1 & $<0.001$ & 1.608 & 1.254 & 2.062 & 1.15 & 3.52 \\
\hline (c) CABG from Medication & & & & & & & Medication & CABG \\
\hline Gender & 6.612 & 1 & 0.010 & 0.306 & 0.088 & 1.062 & 18 & 7 \\
\hline Hypercholesterolemia & 6.892 & 1 & 0.009 & 0.410 & 0.169 & 0.994 & 45 & 31 \\
\hline Hypertension & 6.773 & 1 & 0.009 & 0.407 & 0.166 & 1.003 & 36 & 22 \\
\hline Cerebral events & 12.419 & 1 & $<0.001$ & 0.067 & 0.004 & 1.018 & 12 & 1 \\
\hline LAD artery disease & 54.350 & 1 & $<0.001$ & 5.396 & 2.553 & 11.407 & 1.56 & 2.64 \\
\hline Circumflex artery disease & 32.294 & 1 & $<0.001$ & 2.647 & 1.617 & 4.333 & 1.30 & 2.30 \\
\hline RCA disease & 7.800 & 1 & 0.005 & 1.599 & 1.024 & 2.495 & 1.80 & 2.27 \\
\hline Number of vessels diseased & 9.668 & 1 & 0.002 & 2.116 & 1.080 & 4.144 & 2.39 & 2.77 \\
\hline Ventricular disease & 7.173 & 1 & 0.007 & 1.737 & 0.998 & 3.023 & 0.56 & 0.93 \\
\hline Angina frequency & 5.824 & 1 & 0.016 & 1.710 & 0.949 & 3.080 & 1.80 & 2.11 \\
\hline Angina functional limitations & 15.130 & 1 & $<0.001$ & 1.660 & 1.168 & 2.358 & 1.29 & 2.15 \\
\hline Dyspnoea functional limitations & 7.619 & 1 & 0.006 & 1.450 & 1.015 & 2.072 & 1.21 & 1.79 \\
\hline SIP: Social behaviour & 7.714 & 1 & 0.005 & 1.175 & 1.008 & 1.371 & 3.96 & 5.32 \\
\hline SIP: Mobility range & 40.346 & 1 & $<0.001$ & 1.683 & 1.299 & 2.181 & 1.00 & 3.52 \\
\hline
\end{tabular}

$=135, \mathrm{p}=0.479)$. Nagelkerke's $\mathrm{R}^{2}=0.464$, indicated the predictor variables were associated reasonably well with the outcome variable. Correct classification of cases moved from $50.4 \%$ to $70.2 \%$ in the final model, with $71.8 \%$ of PTCA patients (i.e. sensitivity) and $68.6 \%$ of medication patients correctly classified (i.e. specificity). The discriminatory power of the model is fairly strong with the c-statistic at 0.849 .

Examination of parameter estimates for categorical predictors in this model indicated that holding other variables constant, (i) having had a previous cardiac intervention increased the odds of PTCA by $\approx 9$ times, and (ii) hypertension increased the odds of being in the medication group by $\approx 4$ times. On the continuous predictors, the odds of being a medication patient increased by $\approx 7 \%$ for each years increase in age. In this instance, an increment of 10 years in age doubled the odds of being in the medication as opposed to PTCA group. Unsurprisingly, greater angina frequency and LAD artery disease both increased the odds of having PTCA. Thus going from 'occasional' angina to 'frequent' angina increased the odds of PTCA by 2.4 times and going from 'mild' to 'moderate' lev- 
els of LAD artery disease increased the odds of PTCA by 2.6 times.

\section{Analyses II: CABG compared to PTCA}

Univariate analyses identified twelve variables as significant predictors of CABG treatment as opposed to PTCA (see Table 3b). A higher level of education, having received previous cardiac interventions, family history of neurological conditions and previous cerebral events were all associated with lowered odds of receiving CABG. Higher scores on the five indices from the angiogram, a family history of IHD and reduced levels of social behaviour and mobility range (SIP-68) resulted in greater odds of CABG. Four of these univariate predictors were retained in the final stepwise multivariate model: education level, previous cardiac intervention, circumflex artery disease and mobility range (see Table $4 \mathrm{~b}$ ). This model was satisfactory with regards to overall model fit, with non-signifi- cant residual variance (deviance $=118.363, \mathrm{df}=138 \mathrm{p}=$ 0.885 ), and Nagelkerke's $R^{2}$ at $57.5 \%$. Classification of cases moved from $50.7 \%$ to $78.5 \%$ correct in the final model; $78.1 \%$ of CABG patients and $78.9 \%$ of PTCA patients were correctly classified. The discriminatory power of the logistic equation was relatively good ( $\mathrm{c}=$ $0.892)$.

The individual parameter estimates indicated that the odds for PTCA increased by $\approx 12$ times if a previous intervention had been undertaken. The continuous predictors revealed that a unit increase in educational level (e.g. going from A-level to graduate standard) almost halved the odds of being in the CABG group. A single additional score on the functional limitation on the SIP mobility range scale increased the odds of CABG by $60 \%$ and moving from 'mild' to 'moderate' levels of circumflex artery disease increased the odds of CABG by $\approx 2$ times.

Table 4: Multivariate analysis comparing pairs of treatment groups

\begin{tabular}{|c|c|c|c|c|c|c|c|c|c|c|}
\hline \multirow[b]{2}{*}{ Parameter } & \multicolumn{7}{|c|}{ Analysis of maximum likelihood estimates } & \multicolumn{3}{|c|}{ odds ratio/p-deviance } \\
\hline & Entry & df & estimate & std. error & Wald $\chi^{2}$ & $\operatorname{Pr}>$ chi square & std. estimate & point estimate & $99 \%$ Wald Cl & $99 \%$ Wald Cl \\
\hline \multicolumn{11}{|l|}{$\begin{array}{l}\text { (a) Medication vs. } \\
\text { PTCA }\end{array}$} \\
\hline Constant & 0 & 1 & 1.131 & 1.454 & 0.606 & 0.437 & . & & & \\
\hline $\begin{array}{l}\text { LAD artery } \\
\text { disease }\end{array}$ & 1 & 1 & 0.970 & 0.240 & 16.374 & $<0.001$ & 0.536 & 2.639 & 1.423 & 4.894 \\
\hline $\begin{array}{l}\text { Previous } \\
\text { intervention }\end{array}$ & 2 & 1 & 1.093 & 0.322 & 11.536 & 0.001 & . & 8.896 & 1.696 & 46.675 \\
\hline Increasing age & 3 & I & -0.065 & 0.021 & 9.364 & 0.002 & -0.397 & 0.937 & 0.887 & 0.990 \\
\hline Hypertension & 4 & I & -0.664 & 0.229 & 8.373 & 0.004 & . & 0.265 & 0.081 & 0.864 \\
\hline $\begin{array}{l}\text { Angina } \\
\text { frequency }\end{array}$ & 5 & 1 & 0.883 & 0.341 & 6.694 & 0.010 & 0.337 & 2.418 & 1.004 & 5.822 \\
\hline \multicolumn{11}{|l|}{$\begin{array}{l}\text { (b) PTCA vs. } \\
\text { CABG }\end{array}$} \\
\hline Intercept & 0 & 1 & -1.889 & 0.710 & 7.091 & 0.008 & . & & & \\
\hline $\begin{array}{l}\text { SIP: Mobility } \\
\text { range }\end{array}$ & 1 & 1 & 0.475 & 0.112 & 17.868 & $<0.001$ & 0.667 & 1.607 & 1.204 & 2.147 \\
\hline $\begin{array}{l}\text { Circumflex } \\
\text { artery disease }\end{array}$ & 2 & 1 & 0.756 & 0.215 & 12.345 & $<0.001$ & 0.490 & 2.128 & 1.223 & 3.704 \\
\hline $\begin{array}{l}\text { Previous } \\
\text { intervention }\end{array}$ & 3 & 1 & -1.246 & 0.337 & 13.674 & $<0.001$ & . & 0.083 & 0.015 & 0.469 \\
\hline $\begin{array}{l}\text { Higher } \\
\text { educational } \\
\text { attainment }\end{array}$ & 4 & 1 & -0.661 & 0.204 & 10.545 & 0.001 & $-0.44 I$ & 0.516 & 0.305 & 0.872 \\
\hline
\end{tabular}

\begin{tabular}{|c|c|c|c|c|c|c|c|c|c|c|}
\hline Intercept & 0 & I & -8.831 & 1.548 & 32.561 & $<0.001$ & . & & & \\
\hline $\begin{array}{l}\text { LAD artery } \\
\text { disease }\end{array}$ & 1 & 1 & 1.616 & 0.373 & 18.804 & $<0.001$ & 0.853 & 5.034 & 1.927 & 13.150 \\
\hline $\begin{array}{l}\text { Previous } \\
\text { cerebral events }\end{array}$ & 2 & 1 & -2.463 & 0.707 & 12.127 & 0.001 & . & 0.007 & 0.000 & 0.277 \\
\hline $\begin{array}{l}\text { SIP: Mobility } \\
\text { range }\end{array}$ & 3 & 1 & 0.620 & 0.162 & $|4.76|$ & $<0.001$ & 0.870 & 1.859 & 1.227 & 2.818 \\
\hline $\begin{array}{l}\text { Circumflex } \\
\text { artery disease }\end{array}$ & 4 & 1 & 1.095 & 0.293 & 13.991 & $<0.001$ & 0.661 & 2.991 & 1.406 & 6.359 \\
\hline
\end{tabular}




\section{Analyses III: CABG compared to medication}

Fourteen predictors, listed in Table $3 c$, were significant in the univariate analyses. Increasing severity within all the angiogram and function related scores significantly increased the likelihood of receiving a CABG. The presence of co-morbidities (hypercholesterolemia, hypertension and previous cerebral events) and being female individually reduced the likelihood of CABG.

The multivariate stepwise analysis of these predictors indicated relatively little residual variance (deviance $=85.691$, $\mathrm{df}=138, \mathrm{p}=0.999)$, a high association between the DV and the predictors (Nagelkerke's $\mathrm{R}^{2}=0.726$ ), and a large proportion of correctly classified cases (84.6\%, up from $51.0 \%$ in the constant only model; sensitivity $=84.9 \%$; specificity $=84.3 \%)$. The discriminatory power of the logistic regression equation was the highest of the three sets $(c=0.944)$.

Having previous cerebral events increased the odds of receiving medication treatment by 142.9 times, (see Table $4 \mathrm{c}$ ). On the continuous measures, the odds of CABG were $\approx 5$ times higher with a one unit increase in LAD artery disease and $\approx 3$ times higher with unit increases in circumflex artery disease severity. Greater mobility range limitations increased the odds of CABG by $\approx 2$ times per unit increase.

\section{Discussion}

This study examined factors that influenced the allocation of patients to different treatments for CAD. Three sets of analyses were conducted comparing actual decisions to undergo: PTCA rather than receive medication, CABG rather than PTCA, and CABG rather than medication.

Univariate analyses revealed a wide range of differentiating factors including, demographic variables, co-morbidities, family histories, symptom reports, functional limitations and angiogram indices (i.e. objective disease reports). While many of these factors are in the treatment guidelines (e.g. co-morbidities, angiogram indices); others are not (e.g. educational level, patient rated functioning or symptom reports), but appear to be, on empirical analysis, implicitly involved in the decision processes, particularly in analyses involving comparisons with PTCA. This indicates that clinicians are undoubtedly aware that multiple factors outside of those explicitly discussed in guidelines (which are usually technical and clinical in nature), should be addressed and guide decision making about treatment modality.

Gender has long been a controversial issue in CAD and much evidence has accumulated regarding poorer outcomes for women [16]. Research investigating, gender differences in patients undergoing evaluation for CAD has found contradictory results but generally suggested that resource utilisation may be lower in women [17-19]. In a large study, Miller et al. [20] did find less coronary angiographies performed in women being assessed for CAD and lower referral for revascularisation in univariate analysis. Although, in this study, females were almost 70\% less likely than males to have CABG when compared to medication, this effect was not robust enough to be retained in the multivariate analysis, suggesting that gender is not a primary concern during this decision process, between the two most disparate treatments. Further, in the other analyses there was no evidence to suggest a bias for males to receive particular interventions. However, these findings must be interpreted in light of the small number of female participants, which may limit the power to find gender effects.

Previous research has shown that mortality increases with age in both CABG and PTCA $[21,22]$. In the present study age was not a significant factor in determining CABG from the other two treatments. The CABG results were unexpected but the increased risks may have been counter-balanced by judgements regarding graft patency and life expectancy. In contrast, an increase of ten years in age nearly doubled the likelihood of medication over PTCA in the multivariate analysis. While this may reflect a natural tendency to perform less invasive procedures in the elderly, it is also important that the PTCA group had significantly higher levels of education in comparison to both medication and CABG groups. One may speculate that the younger more educated individuals may be both more aware of and willing to try newer treatments and possibly more actively involved in their treatment decisions. It is however important that the PTCA group were also distinguished by their greater experience of CAD treatments and possible vicarious knowledge/experience from family members who had a history of IHD. PTCA patients were more likely to have received a previous intervention (mainly PTCA's) than both the medication and CABG patients in both the univariate and multivariate analyses. Given the relatively high rates of restenosis following PTCA, this finding is not unexpected. The previous experiences may have provided patients with clearer expectations upon which to base and participate in the decisions regarding their treatment.

Variables concerning patient history and co-morbidities appeared to appropriately reduce the likelihood of the more interventional treatments. Hypertension had a tendency to increase the odds of remaining on/starting medication. In the PTCA versus medication analysis, this effect was retained in the multivariate analysis. Hypercholesterolemia reduced the likelihood of CABG as opposed to medication, and the effects of a family history of neurological problems reduced the likelihood of CABG from PTCA. Less obviously a family history of IHD, reduced the 
Table 5: Summary findings of univariate and multivariate significant effects

\begin{tabular}{|c|c|c|c|}
\hline Univariate & PTCA from Medication & CABG from PTCA & CABG from Medication \\
\hline Increasing age & $\nabla$ & & \\
\hline Gender & & & $\nabla$ \\
\hline Higher educational attainment & $\boldsymbol{\Delta}$ & $\nabla$ & \\
\hline \multicolumn{4}{|l|}{ mployment } \\
\hline Previous coronary intervention & $\Delta$ & $\boldsymbol{\nabla}$ & \\
\hline Hypercholesterolemia & & & $\nabla$ \\
\hline Hypertension & $\nabla$ & & $\nabla$ \\
\hline \multicolumn{4}{|l|}{ Previous MI } \\
\hline \multicolumn{4}{|l|}{ Diabetes } \\
\hline \multicolumn{4}{|l|}{ Smoking history } \\
\hline Family history: IHD & $\nabla$ & $\Delta$ & \\
\hline Family history: Neurological & & $\nabla$ & \\
\hline \multicolumn{4}{|l|}{ Family history: $\mathrm{MI}$} \\
\hline \multicolumn{4}{|l|}{ Family history: Stroke } \\
\hline Previous cerebral events & & $\nabla$ & $\nabla$ \\
\hline \multicolumn{4}{|l|}{ Arthritis/Rheumatism } \\
\hline \multicolumn{4}{|l|}{ Respiratory } \\
\hline \multicolumn{4}{|l|}{ Renal complications } \\
\hline \multicolumn{4}{|l|}{ GI tract problems } \\
\hline \multicolumn{4}{|l|}{ Peripheral vascular disease } \\
\hline \multicolumn{4}{|l|}{ Thyroid problems } \\
\hline Varicose veins & & & \\
\hline
\end{tabular}

LAD artery disease
Circumflex artery disease
RCA disease
Number of vessels diseased
Ventricular disease

Angina frequency

Dyspnoea frequency

Non-cardiac symptoms

Angina functional limitations

Dyspnoea functional limitations

SIP: Social behaviour

SIP: Mobility range

SIP: Emotional stability

SIP: Somatic autonomy

SIP: Mobility control

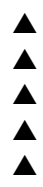

$\Delta$

$\boldsymbol{\Delta}$

$\Delta$

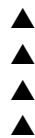

\begin{tabular}{lccc}
\hline Multivariate & PTCA from Medication -5 & CABG from PTCA -4 & CABG from Medication - 4 \\
\hline $\begin{array}{l}\text { Increasing age } \\
\text { Higher educational attainment }\end{array}$ & $\mathbf{\nabla 3}$ & $\mathbf{\nabla 4}$ & $\mathbf{\nabla 3}$ \\
\hline $\begin{array}{l}\text { Previous coronary intervention } \\
\text { Hypertension }\end{array}$ & $\mathbf{\Delta 2}$ &
\end{tabular}

Previous cerebral events

LAD artery disease

Circumflex artery disease

Angina frequency

SIP: Mobility range
$\Delta \mathbf{I}$

$\Delta \mathbf{5}$

$\boldsymbol{\Delta}=$ increase in odds; $\boldsymbol{\nabla}=$ decrease in odds; numerals indicate order of entry during the stepwise procedure 
odds of PTCA when compared to both medication and CABG. However, none of these findings were retained in the multivariate analyses.

The measures from the angiogram reports demonstrated a strikingly consistent effect in predicting the likelihood of CABG, as would be expected and is supportive of the guidelines. Within the univariate analysis, all five of the measures (levels of LAD, circumflex and right coronary artery disease, the number of disease arteries and the level of ventricular disease) increased the likelihood of CABG. The per unit increase in these measures led to a multiplication in odds of CABG ranging from 1.6 to 5.4 times in the medication versus CABG analyses, and between 1.5 and 2.5 times for the PTCA versus CABG analysis. For the most part, the odds ratios determining CABG from medication were larger than those determining CABG from PTCA, as would be expected. The other significant result from the univariate analyses of the angiogram data showed that increasing LAD artery disease predicts a greater likelihood of PTCA from medication; a finding consistent with treatment guidelines.

The multivariate analyses helped determine which of the angiogram measures was the most relevant to the treatment decision process. The pattern of results indicated that with increased LAD disease, medication was not considered a sufficient treatment; the likelihood of PTCA or CABG was increased. Moving from mild to moderate LAD disease increased the odds of PTCA from medication by $\approx$ 2.6 times, and the odds of CABG from medication by approximately twice this (5 times). Increasing LAD disease did not significantly alter the odds of CABG in comparison to PTCA.

Increasing levels of disease in the circumflex artery increased the likelihood that CABG would be the chosen option. A move from mild to moderate levels of circumflex artery disease more than doubled the odds of CABG from PTCA and almost trebled the odds of CABG as opposed to medication. Interestingly, the number of diseased arteries, which is often an indication for CABG rather than PTCA [6], was not retained in the multivariate model. These results are reassuring in that they confirm the important role of the angiogram reports when deciding whether to proceed with interventional strategies, in particular CABG.

Patient reports of the frequency of their symptoms, and the manner in which their poor health is affecting their lives, are the customary way in which patients present the condition to their health care professionals. High levels of angina symptom reports and greater functional limitations increased the likelihood of receiving either of the two more invasive procedures. They did not, however, sig- nificantly affect the decision between PTCA and CABG. The symptom reports that appeared to drive the decisions were specific and non-cardiac symptoms did not affect the treatment decision. In the multivariate analysis, the frequency of angina was retained when comparing PTCA to medication, such that moving from occasional to frequent angina increased the odds of PTCA by $\approx 2.4$ times. The finding that only one symptom measure entered each multivariate analysis is likely to be due to the degree of correlation between the various symptom report measures (range from 0.731 to 0.225 all $p<0.001$ ).

Reports of restrictions on activities of daily living were also found to influence treatment decisions. Specifically, greater limitations in social behaviours and mobility increased the likelihood of receiving CABG, from both PTCA and medication. When entered into the multivariate analysis, the illnesses effect on mobility was the only measure of activities of daily living that was retained; increasing the odds of CABG from medication and PTCA by $80 \%$ and $60 \%$ respectively, for a unit increase in mobility range problems. This suggests that mobility restrictions are an important trigger to provoke more invasive treatments by cardiologists.

Overall each category of information was represented within the multivariate analyses, although all the categories were not represented in each of the analyses. This suggests that all types of information are being considered during the decision processes, although some may only be evident when examined within specific treatment comparisons. Two distinct patterns of outcomes can be discerned from the cumulative results of the three sets of analyses. First, the increased likelihood of CABG was primarily based on reports of greater objective disease (angiograms) and functional limitations. Second, the data suggests that decisions concerning PTCA can be interpreted to be driven by patient characteristics which may reflect the influence of patient preferences although these were not directly measured in this study. The findings reveal that treatment decisions are subject to a range of different influences and not simply guided by protocols.

It must be noted that treatment modalities/technologies and the guiding protocols are changing constantly. The management of coronary disease has changed over the period since the participants for this study were recruited (e.g. as many as three times more percutaneous coronary interventions are now done in the UK). However, although the manner in which factors are addressed in allocating patients to treatments modalities during the decision making process has altered in guidelines, the specific factors that are considered have not changed significantly in the recent updates $[4,6]$. 
Potential limitations to this study include the relatively small sample of participants, which were predominately male and drawn from a limited geographical region, taken from a small pool of cardiologists; resulting in a highly selective patient population (with low incidences of some co-morbidities). Each of these factors may limit the generalisability of results (e.g. the small number of female participants may have precluded finding significant gender effects). A larger multi-centre study, with stratified sampling may help to resolve some of these issues, and it is acknowledged that both geographical and individual consultant factors may confound treatment decisions. For instance there may be a proclivity to perform interventional cardiology in certain regions and/or that individual cardiologists may be referred particular patients, and have preferences for particular treatments [23]. More detailed analysis of such factors with a larger study sample may help to unravel how such factors confound treatment decision processes.

Additionally, a limitation of observational studies, such as the current one, is that they cannot exclude as explanations other unmeasured factors [11]. It may be necessary to conduct further studies to see how recent changes in treatment and technology effects decision making. This study provides both a basis for this and evidence that treatment decisions are subject to a range of different influences including those in protocols.

\section{Conclusion}

Although there are a number of clinical guidelines for deciding between the treatments available for CAD, this study shows that variables beyond those in these protocols, such as patient reports of disability and patient characteristics, may also be important variables when therapy decisions are undertaken. The role of these factors and their relationship and interactions with factors emphasised in the guidelines require further investigation to delineate the processes involved in treatment decisions for CAD.
Abbreviations
ANOVA - analysis of variance
CABG - coronary artery bypass grafting surgery
CAD - coronary artery disease
IHD - ischemic heart disease
LAD - left anterior descending
MCAR - missing completely at random
PTCA - percutaneous transluminal coronary angioplasty

RCA - right coronary artery

SIP - sickness impact profile

\section{Competing interests}

The author(s) declare that they have no competing interests.

\section{Authors' contributions}

SPH \& SPN contributed to the development and design of the study

SPH, SPN, JAH \& SS contributed to the analysis of the data

All authors contributed to the writing and reviewing of the manuscript

\section{Acknowledgements}

The first author was partially supported by a Graduate School Scholarship from University College London. We would also like to thank the constructive comments of the reviewers of the paper.

\section{References}

I. Petersen S, Peto V, Rayner M: Coronary heart disease statistics BHF: London; 2005.

2. Department of Health. Hospital Episodes Statistics 2005 [http://www.dh.gov.uk/PublicationsAndStatistics/Publications/Publica tionsPolicyAndGuidance/PublicationsPolicyAndGuidanceArticle/fs/ en?CONTENT ID=4105545\&chk=znAfqu]. I-4-2005.

3. Eagle KA, Guyton RA, Davidoff R, Ewy GA, Fonger J, Gardner TJ, Gott JP, Herrmann HC, Marlow RA, Nugent W, O'Connor GT, Orszulak TA, Rieselbach RE, Winters WL, Yusuf S: ACC/AHA guidelines for coronary artery bypass graft surgery: executive summary and recommendations: a report of the American College of Cardiology/American Heart Association Task Force on Practice Guidelines (Committee to Revise the 199 I Guidelines for Coronary Artery Bypass Graft Surgery). Circulation 1999, 100: 1464-1480.

4. Eagle KA, Guyton RA, Davidoff R, Edwards FH, Ewy GA, Gardner T], Hart JC, Herrmann HC, Hillis LD, Hutter AM Jr, Lytle BW, Marlow RA, Nugent WC, Orszulak TA: ACC/AHA 2004 guideline update for coronary artery bypass graft surgery: summary article: a report of the American College of Cardiologyl American Heart Association Task Force on Practice Guidelines (Committee to Update the 1999 Guidelines on Coronary Artery Bypass Graft Surgery). J Am Coll Cardiol 2004, 44:1146-II54.

5. Smith SC Jr, Dove JT, Jacobs AK, Kennedy JW, Kereiakes D, Kern MJ, Kuntz RE, Popma JJ, Schaff HV, Williams DO: ACC/AHA guidelines for percutaneous coronary intervention: executive summary and recommendations: a report of the American College of Cardiology/American Heart Association Task Force on Practice Guidelines (Committee to Revise the 1993 Guidelines for Percutaneous Transluminal Coronary Angioplasty). J Am Coll Cardiol 200I, 37:2215-2238.

6. Smith SC Jr, Feldman TE, Hirshfeld JW Jr, Jacobs AK, Kern MJ, King SB III, Morrison DA, O'Neill WW, Schaff HV, Whitlow PL, Williams DO: ACC/AHA/SCAI 2005 guideline update for percutaneous coronary intervention-summary article: a report of the American College of Cardiology/American Heart Association Task Force on Practice Guidelines (ACC/AHA/SCAI Writing Committee to Update the 200I Guidelines for Percutaneous Coronary Intervention). Catheter Cardiovasc Interv 2006, 67:87-II2.

7. Silber S, Albertsson P, Aviles FF, Camici PG, Colombo A, Hamm C, Jorgensen E, Marco J, Nordrehaug JE, Ruzyllo W, Urban P, Stone GW, Wijns W: Guidelines for percutaneous coronary interventions. The Task Force for Percutaneous Coronary Interven- 
tions of the European Society of Cardiology. Eur Heart ] 2005, 26:804-847.

8. Ryan TJ, Antman EM, Brooks NH, Califf RM, Hillis LD, Hiratzka LF, Rapaport E, Riegel B, Russell RO, Smith EE III, Weaver WD: 1999 update: ACCIAHA guidelines for the management of patients with acute myocardial infarction: executive summary and recommendations: a report of the American College of Cardiology/American Heart Association Task Force on Practice Guidelines (Committee on Management of Acute Myocardial Infarction). Circulation 1999, 100:1016-1030.

9. Ziskind AA, Lauer MA, Bishop G, Vogel RA: Assessing the appropriateness of coronary revascularization: The University of Maryland Revascularization Appropriateness Score (RAS) and its comparison to RAND Expert Panel Ratings and American College of Cardiology American Heart Association guidelines with regard to assigned appropriateness rating and ability to predict outcome. Clin Cardiol 1999, 22:67-76.

10. Leape LL, Weissman JS, Schneider EC, Piana RN, Gatsonis C, Epstein AM: Adherence to practice guidelines: the role of specialty society guidelines. Am Heart J 2003, 145:19-26.

II. Hemingway H, Crook AM, Feder G, Banerjee S, Dawson JR, Magee P, Philpott S, Sanders J, Wood A, Timmis AD: Underuse of coronary revascularization procedures in patients considered appropriate candidates for revascularization. New Eng J Med 200I, 344:645-654.

12. Feinstein AR, Fisher MB, Pigeon JG: Changes in Dyspnea-Fatigue Ratings As Indicators of Quality of Life in the Treatment of Congestive Heart-Failure. Am / Cardiol 1989, 64:50-55.

13. Debruin AF, Diederiks JPM, Dewitte LP, Stevens FCJ, Philipsen H: The Development of A Short Generic Version of the Sickness Impact Profile. J Clin Epidemiol 1994, 47:407-4I8.

14. Rubin DB: Multiple Imputation for Nonresponse in Surveys New York: John Wiley \& Sons; 1987.

15. Schafer JL: NORM: Multiple imputation of incomplete multivariate data under the normal model, Version 2.03. Software for Windows 95/98/NT. 2000 [http://www.stat/psu.edu/ jils/mis oftwa.html].

16. East MA, Jollis JG, Nelson CL, Marks D, Peterson ED: The influence of left ventricular hypertrophy on survival in patients with coronary artery disease: Do race and gender matter? I $\mathrm{Am}$ Coll Cardiol 2003, 41:949-954.

17. Battleman DS, Callahan M: Gender differences in utilization of exercise treadmill testing: a claims-based analysis. J Healthc Qual 200I, 23:38-4I.

18. Roger VL, Farkouh ME, Weston SA, Reeder GS, Jacobsen SJ, Zinsmeister AR, Yawn BP, Kopecky SL, Gabriel SE: Sex differences in evaluation and outcome of unstable angina. JAMA 2000, 283:646-652.

19. Leape LL, Hilborne LH, Bell R, Kamberg C, Brook RH: Underuse of cardiac procedures: Do women, ethnic minorities, and the uninsured fail to receive needed revascularization? Ann Intern Med 1999, 130:183-192.

20. Miller TD, Roger VL, Hodge DO, Hopfenspirger MR, Bailey KR, Gibbons RJ: Gender differences and temporal trends in clinical characteristics, stress test results and use of invasive procedures in patients undergoing evaluation for coronary artery disease. J Am Coll Cardiol 200I, 38:690-697.

21. Alexander KP, Anstrom KJ, Muhlbaier LH, Grosswald RD, Smith PK Jones RH, Peterson ED: Outcomes of cardiac surgery in patients age $>=\mathbf{8 0}$ years: Results from the National Cardiovascular Network. J Am Coll Cardiol 2000, 35:73I-738.

22. Batchelor WB, Anstrom KJ, Muhlbaier LH, Grosswald R, Weintraub WS, O'Neill WW, Peterson ED: Contemporary outcome trends in the elderly undergoing percutaneous coronary interventions: Results in 7,472 octogenarians. J Am Coll of Cardiol 2000, 36:723-730.

23. Di Salvo TT, Paul SD, Lloyd-Jones D, Smith AJ, Villarreal-Levy G, Bamezai V, Hussain SI, Eagle KA, O'Gara PT: Care of acute myocardial infarction by noninvasive and invasive cardiologists: procedure use, cost and outcome. J Am Coll Cardiol 1996, 27:262-269.

\section{Pre-publication history}

The pre-publication history for this paper can be accessed here: http://www.biomedcentral.com/1471-2261/6/31/prepub

Publish with Biomed Central and every scientist can read your work free of charge

"BioMed Central will be the most significant development for disseminating the results of biomedical research in our lifetime. "

Sir Paul Nurse, Cancer Research UK

Your research papers will be:

- available free of charge to the entire biomedical community

- peer reviewed and published immediately upon acceptance

- cited in PubMed and archived on PubMed Central

- yours - you keep the copyright

Submit your manuscript here:

http://www.biomedcentral.com/info/publishing_adv.asp
BioMedcentral 